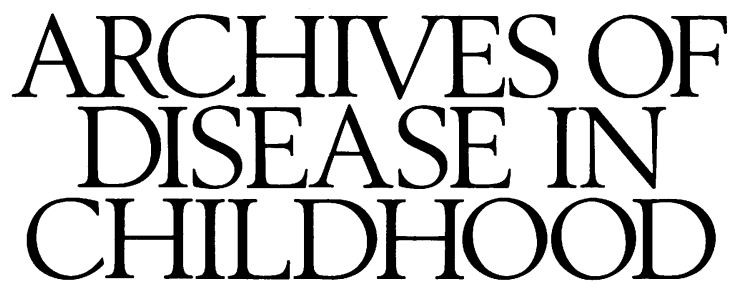

The fournal of the British Paediatric Association

\title{
Annotations
}

\section{Does cytomegalovirus present an occupational risk?}

When cytomegalovirus (CMV) infection is raised as an occupational health issue, this usually relates to an immediate situation. For example, a child with congenital cytomegalovirus infection has been admitted to a nursery; what advice should be given to the pregnant nursery assistant? A nurse looking after an infant with symptomatic congenital cytomegalovirus in a special care baby. unit realises she is pregnant; should she be advised to have a blood test to determine her cytomegalovirus status?

In healthy individuals cytomegalovirus infection is usually asymptomatic and, as a result, few people are aware of their cytomegalovirus status. In the UK seropositivity rises from around $20 \%$ in early childhood to over $80 \%$ in late middle age. ${ }^{2}$ Once acquired, the virus becomes latent and, like other herpes viruses, may reactivate throughout life. Both the primary infection and any recurrences are likely to result in virus shedding in the urine and other body fluids.

Infection may have serious consequences for individuals who are immunocompromised, however, for instance those undergoing cancer treatment or having transplant surgery, people with HIV infection, and very premature infants. Infants born with congenital cytomegalovirus infection are also at risk, overall about $10 \%$ of them will have long term sequelae attributable to cytomegalovirus, the most frequent defect being sensorineural hearing loss; approximately half the affected children have severe physical and/or intellectual handicap. ${ }^{3}$ Nevertheless, about $90 \%$ of congenitally infected babies will develop normally.

\section{Transmission of infection}

Congenital cytomegalovirus infection can result from both primary and recurrent maternal infection at any stage of pregnancy. ${ }^{4}$ While congenital infection is comparatively rare, occurring in approximately $3 / 1000$ births in the UK, cytomegalovirus is commonly transmitted from a seropositive mother to her baby at the time of delivery or in the early months of life. About one child in five has acquired infection by 12 months and virus excretion may continue for several years in normal healthy children. ${ }^{15}$

In a population where many people escape cytomegalovirus in early childhood, sexual transmission plays an important part in adult acquisition of infection. Like other sexually transmitted diseases, cytomegalovirus seropositivity is associated with the number of sexual partners and inversely with the use of barrier contraception. ${ }^{6}$ Blood transfusion and organ transplantation are also effective, but less common, routes of transmission.

Family studies have shown that when a young child or baby acquires the infection, for example from a blood transfusion, most other susceptible household contacts serocon- vert over the next few months. However, this is not an airborne infection and casual contact presents little opportunity for transmission of the virus. Close, prolonged, and intimate contact such as that which occurs between children and adults in a family is required.

\section{Health care settings}

Many hospital patients excrete cytomegalovirus at some time during their hospital stay. For example, a substantial proportion of children on a paediatric ward are likely to be shedding the virus in their urine, it is common for seropositive women to excrete the virus from the cervix, and cytomegalovirus disease is a well recognised complication in immunosuppressed individuals, such as those with HIV infection. In a Minnesota hospital $12 \%$ of patients on the acute care renal ward shed cytomegalovirus during their hospital admission. ${ }^{7}$

Many health professionals with occupational exposure to cytomegalovirus are women of childbearing age. There have been several instances where an individual patient has been thought to be the occupational source of cytomegalovirus infection in a pregnant nurse or doctor. However, restriction enzyme analysis of cytomegalovirus DNA from the suspected source and the congenitally infected infant, or the products of conception, have shown the virus strains to be dissimilar and have excluded the suspected source of infection in all the published case reports to date. ${ }^{89}$

Many epidemiological studies have investigated cytomegalovirus seroprevalence and seroconversion rates among health professionals. These studies suggest that rates of acquisition of the virus are similar to those found in the general population, and that hospital staff are not at increased risk of acquiring cytomegalovirus infection, even if they are working in paediatric, neonatal intensive care, or acute renal units. ${ }^{710}$ It is likely that this reflects the protective nature of normal infection control procedures within the health care setting.

\section{Day care settings}

Three recent studies in the US have identified unexpectedly high rates of seroconversion among daycare workers. ${ }^{11-13}$ Differences in the organisation of daycare, including the number of children catered for, their age mix and social background, as well as different patterns of acquisition of cytomegalovirus infection in different communities, may explain why these findings have not been reported elsewhere. There is no evidence to suggest that cytomegalovirus infection is a particular problem in British nurseries or playgroups. 
Nevertheless, it is reasonable to assume that nursery staff do not routinely practise the kind of infection control procedures carried out in hospitals, but deal with children more as one would at home. Many of these workers come into daily contact with large numbers of young children, some of whom will be excreting cytomegalovirus in saliva or urine.

The growing awareness that nursery staff may be exposed to many infections, including hepatitis B and HIV, has highlighted the need to ensure that high standards of hygiene are maintained within the day nursery. In this context, the overall standard of hygiene practised within a day nursery should be more than adequate to prevent the acquisition of cytomegalovirus. Health education about infections in the community, including cytomegalovirus, should include information about their modes of transmission and means of control, and be a fundamental element in the training of nursery staff and other professionals working with young children in the community. As far as cytomegalovirus is concerned, particular attention should be paid to avoiding the exchange of saliva, and hands should be washed after changing nappies or helping children to use the toilet.

\section{Individual concerns}

To return to our opening example: the congenitally infected child in a day nursery is just one child likely to be excreting virus intermittently alongside many others who will not be recognised. The pregnant nursery assistant can continue to care for that child along with all the others, and this can include changing nappies or helping the child use the toilet, as long as the caregiver washes her hands thoroughly afterwards. She can also cuddle and comfort the child, although practices which might involve the exchange of body fluids, such as exchanging wet kisses or half eaten food should be avoided, not just with a child known to be excreting cytomegalovirus, but with any child.

The pregnant paediatric nurse looking after the infant with congenital cytomegalovirus should not automatically be advised to have blood tests to ascertain her cytomegalovirus status. Under normal circumstances she is extremely unlikely to have acquired an infection from this source. If a recent or current infection were to be diagnosed, invasive investigations could determine whether the fetus was infected (about two in three escape infection) but not whether it was the one in 10 who would be damaged. ${ }^{14}$ Cytomegalovirus infection in pregnancy can cause damage at any stage of gestation and for most women who seroconvert in pregnancy the information is likely to come too late for termination to be a realistic option. There is no treatment to offer.

\section{Recommendations}

- Children known to be excreting cytomegalovirus should not be excluded from nurseries or any other situation. They constitute only a small proportion of infectious children, most of whom are asymptomatic and unrecognised. There is no reason to screen patients or children routinely for cytomegalovirus excretion. A significant proportion will be shedding virus, and the absence of virus on one occasion does not exclude infection as shedding is intermittent. The assumption must be that anyone might be infectious.

- Occupational exposure to cytomegalovirus infection is only one of many reasons for encouraging adherence to infection control procedures in hospital settings.

- In nurseries and other community settings providing for the care of young chidren, proper facilities for reason- able hygiene should be ensured, and their use encouraged. It is reasonable to suggest that pregnant women who work with young children should minimise the kind of contact that might involve the transfer of saliva, and should wash their hands thoroughly after changing nappies or carrying out other intimate tasks. There must be adequate handwashing facilities for staff, washbasins should be located near to where nappies are changed, and there should be proper arrangements for the disposal of used nappies.

- Neither nursery nor hospital staff should be routinely screened for cytomegalovirus status. This is a common infection, there is no clear advice to be given on the basis of results from a screening exercise, and there is no vaccine available. Staff should receive good health education about cytomegalovirus and other infections they are likely to encounter, and understand the reasons for practising good personal hygiene.

- Everyone is exposed to asymptomatic cytomegalovirus excretors not only at work but also in their social and family life, where intimate contact and transmission of infection is far more likely than at work. Nevertheless, individual pregnant staff may have particular anxieties which should be managed sensitively.

Detailed guidelines have been drawn up by various groups working in North America, ${ }^{15-18}$ similiar to those outlined here. However, further research is required to establish whether or not transmission of cytomegalovirus infection to day nursery staff does occur in the UK.

PAT TOOKEY

Epidemiology and Biostatistics,

Institute of Child Health.

30 Guilford Street, London WCIN IEH

CATHERINE S PECKHAM

1 Peckham CS, Johnson C, Ades A, Pearl K, Chin KS. Early acquisition of cytomegalovirus infection. Arch Dis Child 1987;62:780-5.

2 Yarnell JWG, Milbank JE, Munro JA, Howells CHL. The prevalence of cytomegalovirus antibody in women: an epidemiological study from South cytomegalovirus antibody in women: an
Wales. Public Health 1982;96:251-5.

3 Best JM. Congenital cytomegalovirus infection. BMF 1987;294:1440-1.

4 Peckham CS, Coleman JC, Hurley R, Chin KS, Henderson K, Preece PM. Cytomegalovirus infection in pregnancy: preliminary findings from a prospective study. Lancet 1983;i:1352-5.

5 Pass RF, Hutto $C$. Group day care and cytomegaloviral infections of mothers and children. Rev Infect Dis 1986;8:599-605.

6 Collier AC, Handsfield HH, Roberts PL, et al. Cytomegalovirus infection in women attending a sexually transmitted disease clinic. $\mathcal{F}$ Infect Dis 1990 162:46-51.

7 Balfour CL, Balfour HH. Cytomegalovirus is not an occupational risk for nurses in renal transplant and neonatal units. $\mathcal{F} A M A \quad 1986 ; 256: 1909-14$

8 Peckham CS, Garrett AJ, Chin KS, Preece PM, Nelson DB, Warren DE. Restriction enzyme analysis of cytomegalovirus DNA to study transmission of infection. F Clin Pathol 1986;39:318-24.

9 Hokeberg I, Grillner L, Reisenfeld T. No evidence of hospital-acquired cytomegalovirus infection in a pregnant pediatric nurse using restriction endonuclease analysis. Pediatr Infect Dis $\mathcal{f} 1988 ; 7: 812-4$.

10 Balcarek KB, Bagley R, Cloud GA, Pass RF. Cytomegalovirus infection among employees of a children's hospital: no evidence for increased risk among employees of a children's hospital: no evidence

11 Murph JR, Baron JC, Kice Brown C, Ebelhack CL, Bale JF. The occupa Murph JR, Baron JC, Kice Brown C, Ebelhack CL, Bale JF. The occupa-
tional risk of CMV infection among day-care providers. JAMA 1991;265: tional risk

12 Pass RF, Hutto C, Dee Lyon M, Cloud G. Increased rate of cytomegalovirus infection among day care centre workers. Pediatr Infect Dis $\mathcal{J}$ 1990;9:

13 Adler SP. Cytomegalovirus and child day care: evidence for an increased infection rate among day-care workers. $N$ Engl $\mathcal{f}$ Med 1989;321:1290-6.

14 Peckham CS. Cytomegalovirus in the neonate. $\mathcal{I}$ Antimicrob Chemother 1989 23(E):17-21.

15 Brady MT. Cytomegalovirus infections: occupational risk for health professionals. Am $\mathcal{F}$ Infect Control 1986;14:197-203.

16 Infectious Diseases and Immunization Committee, Canadian Paediatric Society. Cytomegalovirus infection in day-care centres: risks to pregnan women. Can Med Assoc f 1990;142:547-9.

17 Pomeroy C, Englund JA. Cytomegalovirus: epidemiology and infection control. Am Y Infect Control 1987;15:107-19.

18 Onorato IM, Morens DM, Martone WJ, Stansfield SK. Epidemiology of cytomegaloviral infections: recommendations for prevention and control. Rev Infect Dis 1985;7:479-97. 